9th A. Friedmann International Seminar and

3rd Casimir Symposium 2015

International Journal of Modern Physics: Conference Series

Vol. 41 (2016) 1660141 (9 pages)

(C) The Author(s)

DOI: $10.1142 /$ S2010194516601411

\title{
Coherent specular reflection of resonant light from a dense ensemble of motionless point-like scatters in a slab geometry
}

\author{
A. S. Kuraptsev \\ Department of Quantum Electronics, Peter the Great St. Petersburg Polytechnic University, \\ Polytechnicheskaya st. 29, Saint Petersburg, 195251, Russia \\ aleksej-kurapcev@yandex.ru
}

I. M. Sokolov

Department of Theoretical Physics, Peter the Great St. Petersburg Polytechnic University, Polytechnicheskaya st. 29, Saint Petersburg, 195251, Russia ims@is12093.spb.edu

Ya. A. Fofanov

Institute for Analytical Instrumentation, Russian Academy of Sciences, Ivana Chernykh st. 31-33, Saint Petersburg, 198095, Russia yakinvest@ya.ru

Received 17 September 2015

Published 18 March 2016

\begin{abstract}
We analyze resonant light scattering from a dense and disordered ensemble of motionless point-like scatters with uniform (on average) spatial distribution of the density. The average interatomic distance is considered comparable with the resonant wavelength and the mean free path of photon. The inhomogeneity of dipole-dipole interaction near the surface is discussed. Angular distribution of the light scattered from a medium in a slab geometry is calculated. The total reflected light power $P$ and the reflectivity $R$ depending on the optical thickness of a medium $b_{z}$ are analyzed. It is shown that in the case of small optical thickness $\left(b_{z} \ll 1\right) P\left(b_{z}\right) \propto b_{z}^{2}$ and $R\left(b_{z}\right) \propto b_{z}$. With further increasing of the optical thickness we observe oscillations of the dependencies $P\left(b_{z}\right)$ and $R\left(b_{z}\right)$. The attenuation coefficient of these oscillations is discussed.
\end{abstract}

Keywords: Coherent scattering; selective reflection; resonant medium; point-like scatters; dipole-dipole interaction.

PACS numbers: 42.25.Dd, 42.50.Nn, 72.15.Rn

This is an Open Access article published by World Scientific Publishing Company. It is distributed under the terms of the Creative Commons Attribution 4.0 (CC-BY) License. Further distribution of this work is permitted, provided the original work is properly cited. 


\section{Introduction}

The wide range of optical detection methods are based on selective reflection of light from the investigated medium. In the majority of these methods resonant or quasi-resonant light is considered. Among a great variety of resonant media the disordered ensembles of point-like scatters in which motion can be neglected take a special place. This model is used for a description of impurity centers in solids and cold but not degenerate atomic ensembles prepared in a special atomic trap.

One of the key parameters of an atomic ensemble (hereafter we will associate point-like scatters with atoms for brevity) is the density. In the case of dilute ensemble (when the average interatomic distance is much greater than resonant wavelength) the atoms can be considered as independent scatters of electromagnetic waves. Coherent cooperative light scattering from a dilute ensemble can be observed only in forward direction. ${ }^{1,2}$

The interaction of resonant light with dense ensemble has a range of important features. Interatomic dipole-dipole interaction significantly influences the optical characteristics of a medium. ${ }^{3}$ Collective effects cause density-dependent shifts of atomic transition as well as distortion of spectral line shape. ${ }^{4-6}$ The real part of dielectric permittivity of dense atomic ensemble can be negative in some spectral area. $^{7,8}$

Dipole-dipole interaction cause coherent light scattering from dense atomic ensemble in backward as well as specular direction. Note that dipole-dipole interaction manifests itself differently for spatial areas inside the medium and near its surface. ${ }^{9}$ The subsurface region is mostly responsible for the reflected beam generation. In Ref. 10 we proved that resonant specular reflection from dense semi-infinite atomic ensemble is almost coherent but the reflectance can not be described by standard Fresnel equations.

In modern studies dense but thin media have attracted much attention. This interest is connected with some exciting features of the cooperative effects (for instance, see Ref. 11). In this paper we study resonant specular reflection from a plane layer of atomic medium depending on its optical thickness.

\section{Basic Assumptions and Approach}

To describe resonant light scattering from dense atomic ensemble we use the quantum microscopic approach developed in our group previously. ${ }^{12}$ This approach is based on the nonstationary Schrodinger equation for the wave function of the joint system consisting of $N$ motionless atoms and electromagnetic field.

We consider all the atoms to be identical. The ground states is characterized by the angular momentum $J=0$. It is separated by the frequency $\omega_{0}$ from the excited state $J=1$. The excited state has the Zeeman structure so there are three sublevels for each atom which differ by the value of angular momentum projection $m=-1,0,1$. Note that it is very important to take into account the Zeeman structure of excited state because the standard two-level scalar model does not allow 
to describe the effects connected with the vector nature of the electromagnetic field correctly. ${ }^{13}$ These effects strongly influence on the light scattering in the case of dense atomic ensembles.

The Hamiltonian of the joined system is presented as a sum of two operators $H_{0}+V$ where $H_{0}$ is the sum of the Hamiltonians of the free atoms and the free field, and $V$ is the operator of their interaction.

Dipole approximation for the operator of interatomic interaction $V$ is used

$$
V=-\sum_{a} \mathbf{d}^{a} \mathbf{E}\left(\mathbf{r}_{a}\right)
$$

In this equation $\mathbf{d}^{a}$ is the dipole moment of the atom $a$ and $\mathbf{E}\left(\mathbf{r}_{a}\right)$ is the value of the field operator at the location point of this atom. The sum should be taken over all atoms in the ensemble. The wave function is found as an expansion in a set of eigenstates $\{|l\rangle\}$ of the operator $H_{0}$.

We assume the probe radiation to be weak and coherent. In quantum optics it is proved that weak and coherent state can be approximated as a superposition of the vacuum and one-photon states. ${ }^{14}$ This allows us to restrict the total number of quantum states taken into account by the following set:

1) $\psi_{g^{\prime}}=|g, g, \ldots, g\rangle \otimes|v a c\rangle$ the vacuum state (all atoms are in the ground state and electromagnetic field is in the vacuum state)

2) $\psi_{g}=|g, g, \ldots, g\rangle \otimes|\mathbf{k} \alpha\rangle$ the onefold excited states of the field subsystem (all atoms are in the ground state and electromagnetic field is in the one-photon state)

3) $\psi_{e_{a}^{m}}=\left|g, \ldots, g, e_{a}^{m}, g, \ldots, g\right\rangle \otimes|v a c\rangle$ the onefold excited atomic states (one atom $a$ is excited, $m$ indicates the Zeeman sublevel of this atom, all other atoms are in the ground state and electromagnetic field is in the vacuum state)

4) $\psi_{e_{a}^{m} e_{b}^{m^{\prime}}}=\left|g, \ldots, g, e_{a}^{m}, g, \ldots, g, e_{b}^{m^{\prime}}, g, \ldots, g\right\rangle \otimes|\mathbf{k} \alpha\rangle$ the nonresonant states (two excited atoms $a$ and $b$ and one photon in the field subsystem)

The amplitude of state $\psi_{g^{\prime}}$ does not change during the evolution of the system, because transitions to this state from other states taken into account are impossible. The transition from $\psi_{g^{\prime}}$ to any other state is also impossible. Nonresonant states are connected with the Heisenberg's uncertainty relation for time and energy. These states are important in the case of dense atomic ensembles for a correct description of the dipole-dipole interaction at short interatomic distances.

The total set of equations for the amplitudes of states $\psi_{g}, \psi_{e_{a}^{m}}$, and $\psi_{e_{a}^{m} e_{b}^{m^{\prime}}}$ is infinite because of the infinite number of field modes. However, we can pick out the finite subset of $3 N$ equations for the amplitudes of the onefold excited atomic states $b_{e_{a}^{m}}$. In Fourier space it is the set of linear algebraic equations. Its formal solution can be written as follows:

$$
b_{e_{a}^{m}}(\omega)=\sum_{b, m^{\prime}} R_{e_{a}^{m} e_{b}^{m^{\prime}}}(\omega) b_{e_{b}^{m^{\prime}}}^{0}(\omega) .
$$

In this equation $R_{e_{a}^{m} e_{b}^{m \prime}}(\omega)$ is the matrix $3 N \times 3 N$ called resolvent which describes the multiple photon exchange among atoms. For a detailed structure of 
this matrix see Ref. 12. The external field is assumed to be a plane monochromatic wave. It enters the right-hand side of Eq. (2) through the vector $b_{e_{b}^{m}}^{0}(\omega)$

$$
b_{e_{b}^{m^{\prime}}}^{0}(\omega)=-\mathbf{d}_{e_{b}^{m^{\prime}} ; g_{b}} \mathbf{e} / \hbar E_{0} \exp \left(i \mathbf{k}_{0} \mathbf{r}_{b}\right) .
$$

Here $\mathbf{d}_{e_{b}^{m^{\prime}} ; g_{b}}$ is the dipole matrix element for transition from the ground $g$ to the excited $m^{\prime}$ state of atom $b . E_{0}$ is an amplitude of probe radiation, $\mathbf{k}_{0}$ and $\mathbf{e}$ are its wave vector and unit polarization vector, $\mathbf{r}_{b}$ is the radius vector of the atom $b$.

The amplitudes of the onefold excited atomic states given by Eq. (2) allow us to obtain the amplitudes of other quantum states $b_{g}$ and $b_{e_{a}^{m}} e_{b}^{m^{\prime}}$. Then, we obtain the wave function and all the observables. In this paper we focus our attention on the characteristics of scattered radiation, primarily on its angular distribution. The intensity of scattered light is calculated as a sum of individual contributions of randomly distributed atoms so it contains a speckle component. In experiments the radiation averaged over an area of a photodetector and integrated over a definite time interval is measured. Therefore in our calculations we perform multiple averaging of observables over random spatial configurations of atoms by the Monte Carlo method.

The approach employed allows us to consider an atomic ensemble with arbitrary shape and spatial distribution of atomic density. However, in this paper we analyze light reflection from a rectangular slab. Spatial distribution of atoms is considered random but uniform on average. The front boundary has the lengths $l_{x}$ and $l_{y}$ along $x$ and $y$ axes respectively. The quantization axis $z$ is directed perpendicular to the front boundary. The thickness of the slab (along $z$ axis) is $l_{z}$. We consider sufficiently large $l_{x}$ and $l_{y}$ so that the reflectance do not depend on these parameters. Hereafter we consider $l_{x}=100 k_{0}^{-1}$ and $l_{y}=50 k_{0}^{-1}$ in this paper. This problem statement is closed to one from Ref. 10, but in the Ref. 10 we studied semi-infinite medium $\left(l_{z}\right.$ was chosen several times greater than the mean free path of a photon). Now we study the case when the optical thickness of a slab is comparable with 1 . In the next section we analyze the reflectance depending on the optical thickness.

\section{Results and Discussion}

At the beginning, let us to analyze the angular distribution of radiation scattered from dense but optically thin atomic ensemble in a slab geometry. We consider the plane of incidence $X Z$ and the incidence angle $\theta$. The density of atoms is chosen $n=0.05 k_{0}^{3}$. It corresponds to the average interatomic distance $r_{a v}=2.71$ (hereafter in this paper we use the inverse wave number of resonant light $k_{0}^{-1}$ as a unit of length). The probe radiation is assumed to be exactly resonant with free atom transition (i.e. its detuning $\Delta=\omega-\omega_{0}=0$ ), and the optical thickness of the slab is considered $b_{z}=0.2$. We determine the optical thickness $b_{z}$ as a ratio of the thickness of a slab $l_{z}$ to the mean free path of photon $l_{p h}$. The last is connected with the imaginary part of the complex wave number of the wave propagating through the medium by a simple equation $l_{p h}=1 /[2 \operatorname{Im}(k)]$. The complex wave number $k$ 


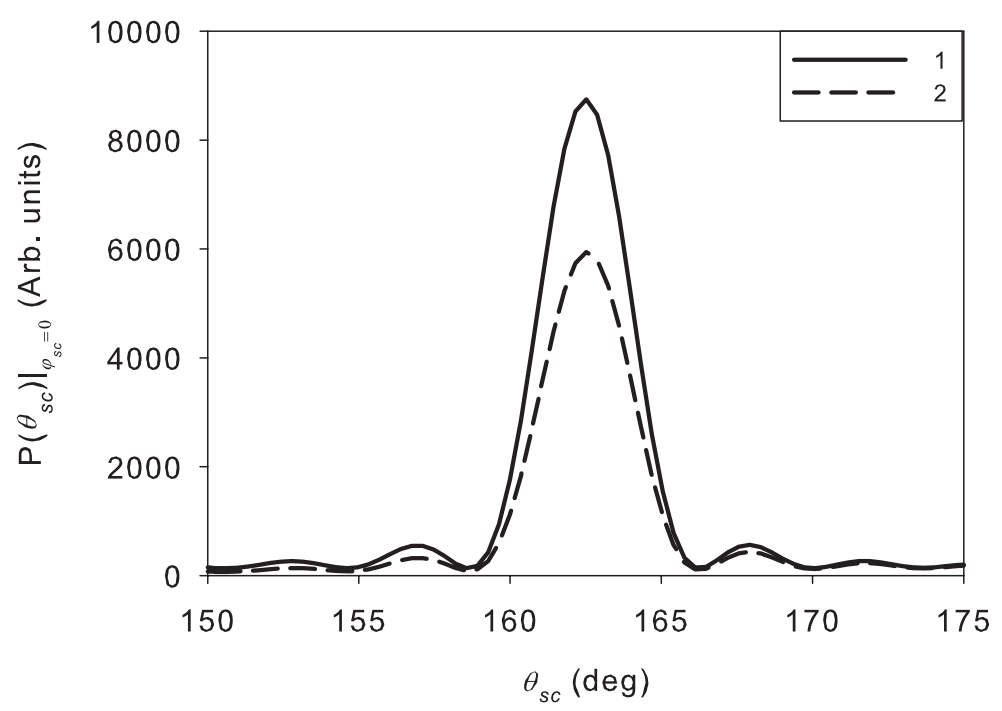

Fig. 1. Angle distribution of the scattered light power at the plane of incidence; $1 s$ polarization, $2 p$ polarization; $n=0.05, \Delta=0, b_{z}=0.2, \theta=17.5^{\circ}$.

was calculated previously, see Ref. 8 for detail. Figure 1 shows the power of light scattered in a unit spherical angle as a function of the polar angle both for $s$ and $p$ polarizations of the probe radiation. It is shown that the maximum of curves corresponds to a well-known reflection law $\left(\theta_{s c}=\pi-\theta_{0}\right)$.

Figure 1 shows the angular density of the total power of scattered radiation. This result is averaged over random spatial positions of atoms by the Monte Carlo method. Both coherent and incoherent scattering can be calculated in this way. In Ref. 15 we have analyzed incoherent diffuse scattering and we have obtained a good agreement with experimental measurements. To calculate the coherent component of the total scattered radiation we average the electric field strength and then we calculate intensity of this component. Our analysis shows that the ratio between the coherent component and the total power of radiation in the reflected signal is close to 1 for the parameters considered here.

The dependence of the scattered radiation power on the azimuthal angle $P\left(\varphi_{s c}\right)$ can be calculated by the similar way. The total power of reflected light $P$ can be obtained as an integral of the angular distribution of scattered light $P\left(\theta_{s c}, \varphi_{s c}\right)$ over the reflected light cone $\Omega_{c}$. $P$ depends on the frequency of probe radiation, its polarization and the angle of incidence, as well as the sizes a sample $l_{x}, l_{y}, l_{z}$. Figure 2 shows the total power of reflected light depending on the optical thickness of a slab.

Dash-dot and dash-dot-dot horizontal lines in Fig. 2 indicate the asymptote of the dependence $P\left(b_{z}\right)$ for $s$ and $p$ polarizations respectively (it is the value of the reflected light power in the case of optically thick slab, $\left.b_{z} \gg 1\right)$. 


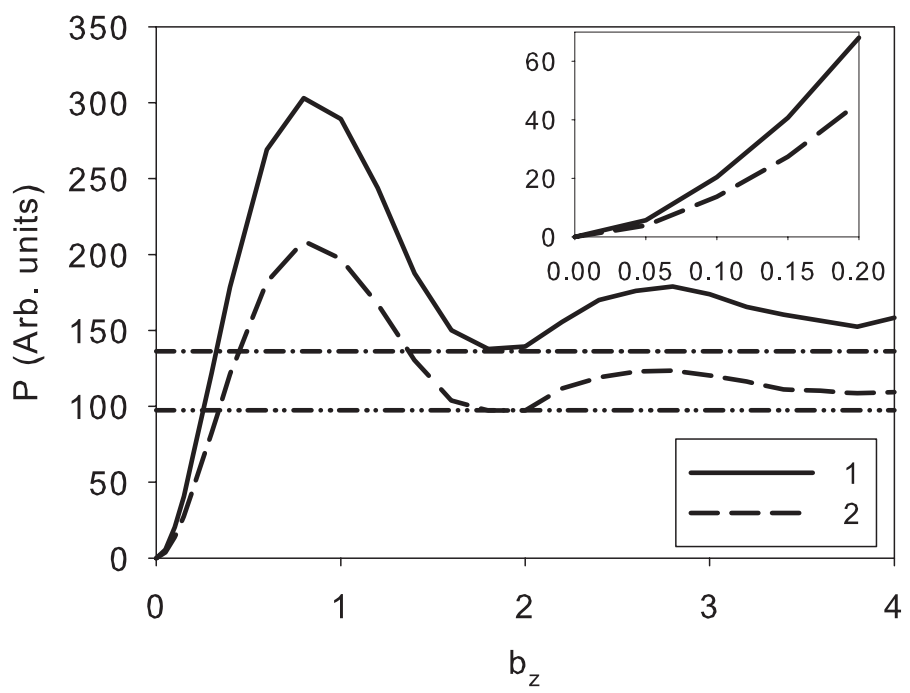

Fig. 2. Dependence of the total reflected light power on the optical thickness of a slab; $1 s$ polarization, $2 p$ polarization; $n=0.05, \Delta=0, \theta=17.5^{\circ}$.

In the inserted plot we can see that for small optical thickness $P\left(b_{z}\right) \propto b_{z}^{2}$. It can be explained by the fact that the reflected light is coherent so its power is proportional to the squared number of atoms. With further increasing of the optical thickness we observe oscillations connected with the interference of waves scattered by different atoms. This interference can be as positive as negative depending on the thickness of a slab. Note that the value of $P$ at the first minimum is approximately equal to its asymptotic value, at the second minimum even more than the asymptotic value. It could seem that this result is in contradiction to one from Ref. 10 where we analyzed the partial contribution of thin layers located at the different distances from the surface to the total power of light reflected from optically thick medium. But it is no real contradiction because in this paper we study light scattering by whole atomic ensemble and do not analyze any parts of a sample like in Ref. 10.

Let us to analyze the attenuation of the oscillations in Fig. 2. The peak amplitude decreases approximately exponentially $\exp \left(-\alpha b_{z} / \cos \theta_{0}\right)$. For $s$ polarization we obtain $\alpha=0.65$ and for $p$ polarization $\alpha=0.69$. Note that $\alpha<1$ for both polarizations. In our opinion, it can be explained by the fact that cooperative effects manifest themselves differently for spatial areas inside the medium and near its surface. In the framework of the approach used here the atomic polarization is calculated taking into account the secondary waves emitted by all the atoms, including the waves which propagate in backward direction.

The reflectivity $R$ is determined as the ratio of the total reflected light power $P$ to the total power of light scattered in all directions $P_{0} . P_{0}$ can be obtained 


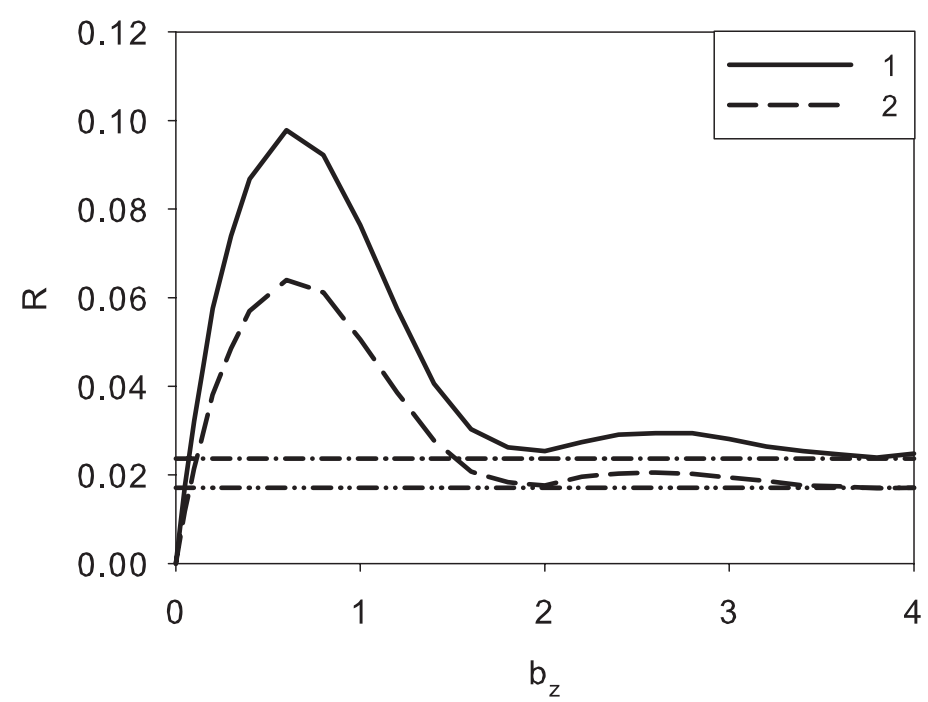

Fig. 3. Dependence of the reflectivity on the optical thickness of a slab; $1 s$ polarization, $2 p$ polarization; $n=0.05, \Delta=0, \theta=17.5^{\circ}$.

using the optical theorem. Figure 3 shows the reflectivity depending on the optical thickness.

In the case of small optical thickness the dependence $R\left(b_{z}\right)$ is close to linear. It is caused by the fact that $P_{0}$ is proportional to the forward scattering amplitude which is proportional to the number of atoms. Thus, $P_{0} \propto b_{z}, P \propto b_{z}^{2}$, so $R=P / P_{0} \propto b_{z}$. With further increasing of the optical thickness we observe oscillations in Fig. 3 which are explained by the interference of waves scattered by different atoms. Dashdot and dash-dot-dot horizontal lines indicate the asymptote like in Fig. 2. The attenuation of the dependence $R\left(b_{z}\right)$ is $\alpha=1.22$ for $s$ polarization and $\alpha=1.26$ for $p$ polarization. It exceeds the attenuation of the dependence $P\left(b_{z}\right)$ shown in the Fig. 2 almost two times. Note that the reflectivity does not depend on the sizes of the front surface $l_{x}$ and $l_{y}$ (with the exception of the case when these sizes are less than or comparable with the wavelength). Thus, the result shown in Fig. 3 is correct for the reflection from a medium which is infinite along $x$ and $y$ directions.

\section{Conclusion}

In this paper we analyze the reflection of resonant light from a disordered atomic ensemble in a slab geometry. The atoms are considered to be motionless. The density of atoms is considered to be large so that the average interatomic distance is comparable with the resonant wavelength and the mean free path of photon. The theoretical approach is based on the nonstationary Schrodinger equation for the 
joint system consisting of atoms and electromagnetic field. Dipole-dipole interatomic interaction is taken into account.

The angular distribution of scattered radiation is studied. Our analysis shows that specularly reflected light is almost coherent. In this paper we focus our attention on the dependence of the reflected light power $P$ and the reflectance $R$ on the optical thickness of a slab $b_{z}$. It is shown that in the case of the optically thin slab $\left(b_{z} \ll 1\right)$ $P \propto b_{z}^{2}$ and $R \propto b_{z}$. With further increasing of $b_{z}$ we observe the oscillations of these dependencies connected with the interference of electromagnetic waves scattered by different atoms (this interference can be as positive as negative depending on the thickness of a slab). We also studied the attenuation of oscillations. It is proved that the attenuation coefficient $\alpha$ of the dependence $P\left(b_{z}\right)$ is definitely less than 1 for both $s$ and $p$ polarizations. It can be explained by the cooperative effects which manifest themselves differently for spatial areas inside the medium and near its surface. The attenuation of the dependence $R\left(b_{z}\right)$ is greater than one of the function $P\left(b_{z}\right)$ almost two times.

In our opinion, microscopic analysis of resonant reflection performed here will be useful for further improvement of optical detection methods based on coherent scattering of resonant light. Especially, the results presented in this paper can be relevant for the case of light reflection from thin films.

The approach used here can be modified for a description of non-resonant light scattering from an ensemble of point-like scatterers. In particular, dense ensembles of magnetic nanoparticles in the presence of a magnetic field are of especial interest. ${ }^{16-18}$ An external magnetic field can be used for the control of the anisotropy of a medium. It is expected that the dependence of the light power reflected from a layer of nanoparticles on its optical thickness should be oscillating. Thereby, the comparison of this dependence with the case of resonant light scattering shown in Fig. 2 can reveal some similarities.

\section{Acknowledgments}

We acknowledge financial support from the Russian Foundation for Basic Research (Grants No. 15-02-01013 and 15-02-08703) as well as the Ministry of Education and Science of the Russian Federation (the Government Task for Higher Education Institutions (basic part) 2014/184, the Government Task for Higher Education Institutions (design part) No. 3.1446.2014K). A.S.K. also appreciates financial support from the RFBR (Grant No. 14-02-31422), the Council for Grants of the President of the Russian Federation, and the nonprofit foundation "Dynasty".

\section{References}

1. C. C. Kwong, T. Yang, M. S. Pramod, K. Pandey, D. Delande, R. Pierrat and D. Wilkowski, Phys. Rev. Lett. 113, 223601 (2014).

2. C. C. Kwong, T. Yang, D. Delande, R. Pierrat and D. Wilkowski, Cooperative emission of a pulse train in an optically thick scattering medium, to appear in Phys. Rev. Lett. 
3. J. Pellegrino, R. Bourgain, S. Jennewein, Y. R. P. Sortais, A. Browaeys, S. D. Jenkins and J. Ruostekoski, Phys. Rev. Lett. 113, 133602 (2014).

4. T. Ido, T. H. Loftus, M. M. Boyd, A. D. Ludlow, K.W. Holman and J. Ye, Phys. Rev. Lett. 94, 153001 (2005).

5. A. S. Kuraptsev and I. M. Sokolov, Phys. Rev. A 90, 012511 (2014).

6. J. Javanainen, J. Ruostekoski, Y. Li and S.-M. Yoo, Phys. Rev. Lett. 112, 113603 (2014).

7. Ya. A. Fofanov, A. S. Kuraptsev and I. M. Sokolov, Opt. Spectrosc. 112, 401 (2012).

8. Ya. A. Fofanov, A. S. Kuraptsev, I. M. Sokolov and M. D. Havey, Phys. Rev. A 84, 053811 (2011).

9. Ya. A. Fofanov, A. S. Kuraptsev, I. M. Sokolov and M. D. Havey, Phys. Rev. A 87, 063839 (2013).

10. A. S. Kuraptsev and I. M. Sokolov, Phys. Rev. A 91, 053822 (2015).

11. J. Keaveney, A. Sargsyan, U. Krohn, I. G. Hughes, D. Sarkisyan and C. S. Adams, Phys. Rev. Lett. 108, 173601 (2012).

12. I. M. Sokolov, D. V. Kupriyanov and M. D. Havey, J. Exp. Theor. Phys. 112, 246 (2011).

13. I. M. Sokolov, D. V. Kupriyanov, R. G. Olave and M. D. Havey, J. Mod. Opt. 57, 1833 (2010).

14. L. Mandel and E. Wolf, Optical Coherence and Quantum Optics (Cambridge University Press, Cambridge, 1995).

15. I. M. Sokolov, A. S. Kuraptsev, D. V. Kupriyanov, M. D. Havey and S. Balik, J. Mod. Opt. 60, 50 (2013).

16. C. Scherer and A. M. Figueiredo Neto, Brazilian Journal of Physics 35, 718 (2005).

17. Ya. A. Fofanov, E. E. Bibik, I. V. Pleshakov and P. M. Agruzov, Precision laser detection of magnetization processes of magnetically ordered substances, in International Conference on Coherent and Nonlinear Optics (Russia, Moscow, 2013), Technical digest, ICONO-08 Ultrafast Phenomena and High-Precision Measurements, p. 12.

18. P. M. Agruzov, I. V. Pleshakov, E. E. Bibik, Ya. A. Fofanov and A. V. Shamrai, Microstructured optical fiber with magnetic fluid cladding: modulation and polarization properties, in International Conference on Coherent and Nonlinear Optics (Russia, Moscow, 2013), Technical digest, ICONO-07 Physics of Metamaterials and Complex Media, p. 22. 